\title{
Optic nerve compression in craniopharyngioma by taut anterior cerebral arteries visualized in frontal and transsphenoidal exposures: illustrative cases
}

\author{
Anna L. Slingerland, BS, Lissa C. Baird, MD, and R. Michael Scott, MD \\ Department of Neurosurgery, Boston Children's Hospital, Boston, Massachusetts
}

BACKGROUND During initial exposure and removal of craniopharyngioma in pediatric patients with severe visual field deficits, the authors have encountered severe deformation of the optic apparatus by taut anterior cerebral arteries as seen during both frontal craniotomy and transsphenoidal exposures.

OBSERVATIONS The authors report two pediatric patients with craniopharyngioma whose severe preoperative visual deficits were associated not only with large suprasellar masses but also with severe optic nerve and chiasm compression by taut anterior cerebral arteries. In each patient, the optic nerves were partially cleft by these vessels' indenting them.

LESSONS The role of a taut anterior cerebral artery complex in compression of the optic apparatus in patients with suprasellar tumors has been reported previously, but the intraoperative images in these two cases dramatically reveal this phenomenon.

https://thejns.org/doi/abs/10.3171/CASE21642

KEYWORDS craniopharyngioma; visual field deficit; anterior cerebral artery compression; operative exposure; optic nerve compression

Visual field deficits and loss of visual acuity are common presenting symptoms in pediatric patients with craniopharyngioma. ${ }^{1,2}$ These deficits are usually believed to be a result of optic nerve compression from tumor mass effect causing ischemic changes in the optic apparatus. ${ }^{3}$ Some authorities have imputed visual symptoms to tight compression from the anterior cerebral arteries (ACAs) from above the tumor, ${ }^{3}$ but the presence of this vascular compression has not been widely recognized.

We report two pediatric patients with craniopharyngiomas who presented with visual field deficits. Intraoperatively, the optic nerves were found to be severely compressed and partially cleft by taut ACAs; in one case, this compression was documented during a subfrontal craniotomy and in the other during transsphenoidal exposure. The dramatic intraoperative images and clinical data are presented.

\section{Illustrative Cases}

\section{Case 1}

A 5-year-old boy presented in 2006 with polydipsia, polyuria, reduced growth rate, and visual loss. His preoperative ophthalmological examination revealed profound visual loss in the left eye and temporal hemianopsia in the right eye. Magnetic resonance imaging (MRI) revealed a cystic sellar and suprasellar mass measuring $3.7 \times 2.1 \times 2.5 \mathrm{~cm}$. The patient was urgently operated on at an outside institution, undergoing transsphenoidal drainage of the cystic component of this lesion and biopsy. The final pathology was consistent with craniopharyngioma, and postoperative imaging showed decompression of the optic chiasm. Follow-up computed tomography 3 months later demonstrated a $2.1 \times$ 2.1-cm recurrence of the suprasellar mass with rim calcifications and recurrent compression of the optic chiasm, and the patient was referred for further management.

At the time of the exposure via right frontal craniotomy, the junction of the right optic tract, chiasm, and nerve was tightly compressed by the ipsilateral right ACA, and, after complete tumor resection, a deep groove created by this arterial compression could be easily observed in the superior surface of the visual apparatus along with a small cleft at the right optic nerve-chiasm-tract junction (Fig. 1). The patient's vision improved slightly in his right eye but remained poor in his left eye. At follow-up 13 years after surgery, there was no tumor recurrence, but he continues to have bitemporal field cuts, with the left side worse than right, and is receiving full hormone replacement therapy. 

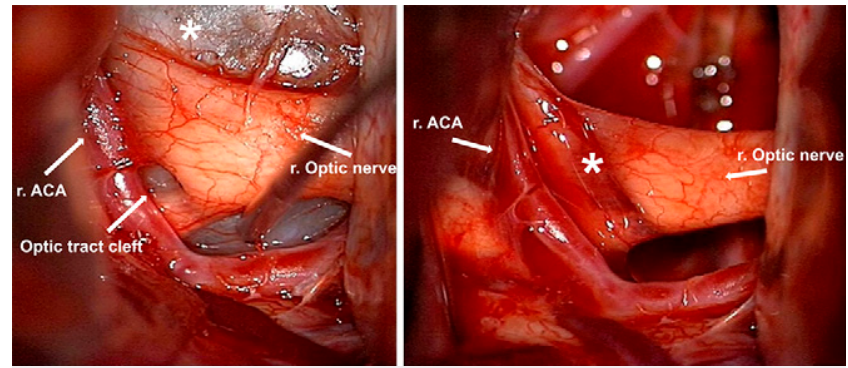

FIG. 1. Left: Initial exposure via right frontal craniotomy in case 1. The tumor can be seen medial to the right optic nerve as well as faintly visualized in the space between the optic nerve and carotid artery. The cleft created in the chiasm-tract junction is also visualized. Right: Operative exposure after tumor excision. The deep grooving of the superior surface of the now relaxed optic chiasm, designated by the asterisk, extends laterally to the cleft seen in the initial exposure.

\section{Case 2}

A 16-year-old girl presented in April 2021 with headache, fatigue, weight loss, polydipsia, polyuria, and amenorrhea. An MRI scan revealed a complex cystic sellar and suprasellar mass measuring $2.4 \times 2 \times 2 \mathrm{~cm}$. Her preoperative ophthalmological examination revealed bitemporal hemianopia and nasal thinning on retinal nerve fiber imaging. She underwent gross total excision of the tumor via a transsphenoidal approach. Intraoperative inspection of the inferior surface of the optic nerves and chiasm after tumor excision revealed deep translucent grooves created by compression of the $A 1$ and $A 2$ branches of the ACA on the superior surface of the optic nerves and chiasm, with complete separation of the left optic nerve fibers in some areas (Fig. 2).

\section{Discussion}

\section{Observations}

There are very few references in the literature to optic nerve compressions by the ACAs in pediatric patients with craniopharyngioma. One case report described an 18-year-old patient who presented with dense left homonymous hemianopsia and right superior temporal quadrantanopsia. ${ }^{4}$ During a gross total resection, the ACA was found to be

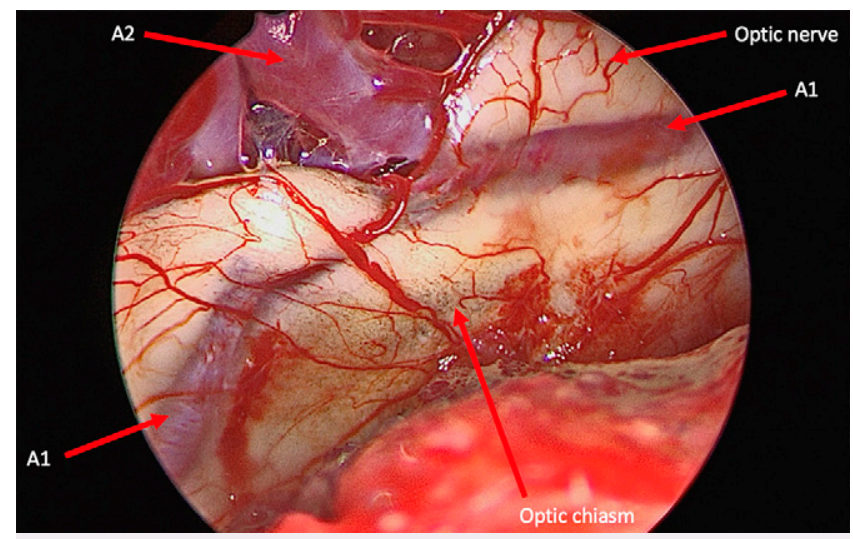

FIG. 2. Exposure in case 2 via transsphenoidal approach after the tumor has been removed, showing the undersurface of the optic apparatus. The A1 branch of the ACA has completely penetrated the left optic nerve, rendering the vessel easily visible through the nerve fibers. pressed against the superior surface of the optic tract. A narrow groove in the right optic tract just posterior to the chiasm was also identified. Additional articles have illustrated grooving on the surface of the optic system from $\mathrm{ACAs}^{5-7}$ but there are no reports of similar compression as seen from below the optic apparatus during a transsphenoidal approach.

We herein document severe compression of the optic apparatus by the ACAs in patients with visual deficits and craniopharyngioma, with intraoperative images recorded both from a superior craniotomy approach in one patient and via a transsphenoidal approach in another. The operative findings left little doubt in the minds of both surgeons (R.M.S., L.C.B.) that the primary factor in each patient's visual symptoms was the compression from above by the ACAs, which partially cleft the optic apparatus in both cases. In both of our cases, a careful review of preoperative imaging studies did not reveal the severity of this compression; it showed only that the ACAs were elevated by the tumor, and we are not certain if any operative maneuvers might improve visual outcomes in patients when this type of compression is found at surgery.

\section{Lessons}

In patients with sellar and suprasellar masses, severe compression of the optic apparatus from above by taut ACAs is a real but underappreciated etiology of preoperative visual deficits.

\section{References}

1. Enayet AER, Atteya MME, Taha $\mathrm{H}$, et al. Management of pediatric craniopharyngioma: 10-year experience from high-flow center. Childs Nerv Syst. 2021;37(2):391-401.

2. Zuccaro G. Radical resection of craniopharyngioma. Childs Nerv Syst. 2005;21(8-9):679-690

3. Yokota H, Yokoyama K, Nishioka T, Iwasaki S, Shimizu H, Kinoshita $\mathrm{S}$. Anterior cerebral artery notching on anterior optic pathways in a child with craniopharyngioma and progressive blindness. Pediatr Neurosurg. 2011:47(5):376-378.

4. Huang PP, Constantini S, Wisoff JH. Etiology of an unusual visual field deficit associated with a craniopharyngioma: case report. Ophthalmologica. 1997;211(4):256-262.

5. Bejjani GK, Cockerham KP, Kennerdell JS, Maroon JC. Visual field deficit caused by vascular compression from a suprasellar meningioma: case report. Neurosurgery. 2002;50(5):1129-1132.

6 . Thomé $\mathrm{C}$, Zevgaridis $\mathrm{D}$. Delayed visual deterioration after pituitary surgery - a review introducing the concept of vascular compression of the optic pathways. Acta Neurochir (Wien). 2004;146(10):1131-1136.

7. Mizrahi CJ, Moscovici S, Dotan S, Spektor S. Optic nerve vascular compression in a patient with a tuberculum sellae meningioma. Case Rep Ophthalmol Med. 2015;2015:681632.

\section{Disclosures}

The authors report no conflict of interest concerning the materials or methods used in this study or the findings specified in this paper.

\section{Author Contributions}

Conception and design: Scott, Baird. Acquisition of data: all authors. Analysis and interpretation of data: all authors. Drafting the article: all authors. Critically revising the article: all authors. Reviewed submitted version of manuscript: all authors. Approved the final version of the manuscript on behalf of all authors: Scott. Administrative/technical/material support: Slingerland. Study supervision: Scott, Baird.

\section{Correspondence}

R. Michael Scott: Boston Children's Hospital, Boston, MA. michael. scott@childrens.harvard.edu. 